\title{
Parameter Estimation of the Linear Phase Correction Model by Mixed-Effects Models
}

\author{
Dominic Noy ${ }^{\mathrm{a}, \mathrm{b}}$, Raquel Menzes ${ }^{\mathrm{a}}$ \\ ${ }^{a}$ Center of Mathematics (CMAT), School of Science, University of Minho, Portugal \\ ${ }^{b}$ Department of Basic Psychology, School of Psychology, University of Minho, Portugal
}

\begin{abstract}
The control of human motor timing is captured by cognitive models that make assumptions about the underlying information processing mechanisms. A paradigm for its inquiry is the Sensorimotor Synchronisation (SMS) task, in which an individual is required to synchronise the movements of an effector, like the finger, with repetitive appearing onsets of an oscillating external event. The Linear Phase Correction model (LPC) is a cognitive model that captures the asynchrony dynamics between the finger taps and the event onsets. It assumes cognitive processes that are modelled as independent random variables (perceptual delays, motor delays, timer intervals).

There exist methods that estimate the model parameters from the asynchronies recorded in SMS tasks. However, while many natural situations show only very short synchronisation periods, the previous methods require long asynchrony sequences to allow for unbiased estimations (see Jacoby, Tishby, Repp, Ahissar \& Keller, 2015b). Also, depending on the task, long records may be hard to obtain experimentally. Moreover, in typical SMS tasks, records are repetitively taken to reduce biases. Yet, by averaging parameter estimates from multiple observations, the existing methods do not most appropriately exploit all available information.
\end{abstract}

Therefore, the present work is a new approach of parameter estimation to

Email addresses: id4256@alunos. uminho.pt (Dominic Noy), rmenezes@math.uminho.pt (Raquel Menzes) 
integrate multiple asynchrony sequences. Based on simulations from the LPC model, we first demonstrate that existing parameter estimation methods are prone to bias when the synchronisation periods become shorter. Second, we present an extended Linear Model (eLM) that integrates multiple sequences within a single model and estimates the model parameters of short sequences with a clear reduction of bias. Finally, by using Mixed-Effects Models (MEM), we show that parameters can also be retrieved robustly when there is betweensequence variability of their expected values.

Since such between-sequence variability is common in experimental and natural settings, we herewith propose a method that increases the applicability of the LPC model. This method is now able to reduce biases due to fatigue or attentional issues, for example, bringing an experimental control that previous methods are unable to perform.

Keywords: Linear Phase Correction Model, Sensorimotor Synchronisation, Parameter Estimation, Mixed-Effects Models

\section{Introduction}

Humans coordinate their movements with nearby moving objects in the environment with a remarkable ease. This requires a highly timed communication of the perception-action systems underpinning the movement control. In or-

5 der to investigate the underlying timing mechanisms, employed by the Central Nervous System (CNS), researchers study participants' attempt to synchronise their movements concurrently with repetitively occurring environmental events. Synchronisation can be understood as a simplified type of coordination because it is constrained in space and time. It is particularly important in activities such as music, sports, and manufacturing. Synchronising movements with a partner was also shown to increase social aspects, such as social attachment and cooperation (Wiltermuth \& Heath, 2009; Valdesolo et al., 2010; Reddish et al., 2013), rapport (Miles et al., 2009), and likability (Launay et al., 2014), and it was traditionally used as a means to enhance self-esteem and obedience 
(Valturio, 1921).

The study of motor synchronisation is mostly focused on effectors like the fingers (Repp, 2005), the forearms (Mörtl et al., 2012), or the feet (van Ulzen et al., 2008) to be timed with external events like auditory metronomes, light displays, or interacting partner movements (Schmidt \& Richardson, 2008; Noy 20 et al., 2017).

A successful synchronisation requires the individual to a) perceive the event onsets; b) perceive one's movement onset; c) compute the asynchrony between both onsets; d) compute the temporal progression of the repeated event series; e) follow all these steps to predict upcoming event onsets.

Based on these perceptual processes, appropriate motor commands can be computed so that the asynchrony-between the movement and the eventbecomes reduced to a minimum (Grush, 2004; Van Der Steen \& Keller, 2013). When the external event is presented with constant temporal intervals (these may also vary slightly), this paradigm is called Sensorimotor Synchronisation

30 (SMS) (Repp, 2005).

There are cognitive models accounting for the empirical findings obtained from SMS tasks. Cognitive models usually use a mathematical representation, formalised as a parametrised system of equations that receives input, for example, sensory cues about the onsets and previous asynchronies and intentions to reduce the asynchrony (Wing \& Kristofferson, 1973; Schulze \& Vorberg, 2002; Jacoby et al., 2015b) and produce output, for example a motor response to reduce the next asynchrony or the actual asynchrony sequence. By solving (or approximating) such systems, its parameters are revealed.

These models can be challenged by comparing their analytical or simulated output - for a given input and set of parameters - with experimental observations. By systematically manipulating the input, it can be validated whether such processes-as postulated by the particular model- in fact underpin the information processing of the CNS.

Because in experiments there are always variables that can neither be manipulated nor controlled-i.e., there is noise within and beyond the CNS-these 
problems are usually approached in a probabilistic manner. Within the framework of probability theory, a model can be defined as a parametric family of probability distributions. The combination of probability distributions (indexed by parameters) determines the distribution of the input and associates a probability of occurrence to each output. Probabilistic models are used to model cognitive abilities. Usually, the challenge is to determine how the input and the output relate to the model parameters in question (Myung, 2003).

In cognitive models of motor synchronisation, the output are the asynchrony dynamics between the onsets of oscillating motion of an individual and the onsets of a repetitively appearing stimulus. The subject of inquiry is the relation of these asynchronies to the parameters of the underlying timing model.

Our scope is a) to give a brief overview of such models, b) present their current parameter estimation approaches and limitations, and c) to introduce a novel approach of parameter estimation. In the introduction, we present the synchronisation models of interest and the most recent parameter estimation method and illustrate that it is biased when certain experimental conditions are not met (i.e., when the asynchrony sequences become shorter). In the main section (methods and results), we present then an extended Linear Model (eLM) revealing superior estimation performance in such conditions. Finally, 65 we present a Mixed-Effects Model, which is a further extension of the eLM, that also accounts for additional intergroup-specific variability. We show that it most accurately and efficiently estimates the model parameters. The main contribution of this work is the finding of robust parameter estimation methods that allow validating the LPC model on more complex empirical observations from movement synchronisation experiments.

\subsection{Timing Models}

\subsubsection{Continuation Tapping}

In order to account for human timing processes, Wing \& Kristofferson (1973) developed a probabilistic cognitive model, which describes the timing behaviour 
intervals. When the intervals are determined by an external metronome that suddenly stops and the individual is required to continue executing the constant movement intervals, this method is called the synchronization-continuation paradigm. Based on the variability of the movement intervals (i.e., the time between two successive taps), Wing \& Kristofferson (1973) proposed the following model $^{1}$ :

$$
I_{j}=C_{j}-D_{j-1}+D_{j}
$$

where $I_{j}$ is the movement interval $j, C_{j}$ is the internal representation of the interval $I_{j}$ (Time Keeper), and $D_{j}$ comprises the perceptual and motor delays. $I_{j}$ is the temporal response interval bounded by two successive taps, which are determined by $C_{j-1}-D_{j-2}+D_{j-1}$ and $C_{j}-D_{j-1}+D_{j}$. In follow-up studies, this was changed to $C_{j}-D_{j-1}+D_{j}$ and $C_{j+1}-D_{j}+D_{j+1}$ (Schulze \& Vorberg, 2002). $C_{j}$ and $D_{j}$ are defined as independent random variables with $C_{j} \sim N V\left(\mu_{C}, \sigma_{C}^{2}\right)$ and $D_{j} \sim N V\left(\mu_{D}, \sigma_{D}^{2}\right)$.

The model in Equation 1 suggests that $\gamma_{I}(1)$ is different of zero due to the simultaneous presence of $D_{j-1}$ and $D_{j}$ at the $j^{\text {th }}$ iteration. Assuming independent random variables, $\gamma_{I}$ is supposed to be zero at larger lags $(>1)$. Taking into account that $D_{j}$ comprises perceptual and motor delays, the serial dependence of $I_{j}$ may reflect the degree of noise (variability) within their respective information processing pathways (Wing \& Kristofferson, 1973).

\subsubsection{Linear Phase Correction Model}

Based on Wing \& Kristofferson (1973)'s model, Schulze \& Vorberg (2002) developed the Linear Phase Correction model (LPC)

\footnotetext{
${ }^{1}$ For the introduction of the existing models and techniques, we used the notation of the original articles. For this reason, notations of the same variables and parameters can vary throughout this work.
} 


$$
A_{n+1}=(1-\alpha) A_{n}+T_{n}+M_{n+1}-M_{n}-C,
$$

where $A_{n}$ is the asynchrony at iteration $n, C$ is a constant metronome interval, $M_{n}$ is the motor delay, and $T_{n}$ is the Time Keeper interval. $M_{n}$ and $T_{n}$ are random variables with $M_{n} \sim N V\left(\mu_{M}, \sigma_{M}^{2}\right)$ and $T_{n} \sim N V\left(\mu_{T}, \sigma_{T}^{2}\right)$

Thus, the LPC describes the temporal behaviour of the observed asynchronies $A_{n+1}$ as a linear combination of the preceding asynchronies $A_{n}$, a cognitive representation of the external event structure $T_{n}$, and the information processing delays within the CNS, $M_{n}$ and $M_{n+1}$.

The LPC received empirical support for its validity (see e.g., Repp, 2005; Torre \& Balasubramaniam, 2009; Zelaznik, Spencer \& Ivry, 2002) and was extended to circumstances in which the base tempo of the metronome changed (i.e., $C_{n}$ as a function of $n$ ) and therefore $\mu_{T}$ had to be adjusted (i.e., period correction) (Repp \& Keller, 2004; Repp, 2001) or when the metronome adjusted its intervals as a function of the individuals' movement dynamics (Repp \& Keller, 2008).

\subsubsection{Parameter Estimation Method}

Traditionally, the parameters of the LPC model in Equation 2 were estimated by the empirical auto-covariance function (acvf) (see Schulze \& Vorberg, 2002).

Yet, it was argued that this estimation is biased when the asynchrony sequences are obtained from SMS tasks with variable metronome intervals. When the temporal intervals changed or phase perturbations occurred-what is common in natural settings - the parameters had to be estimated by fitting the empirical acvf to computer simulations, which is slow and often no unique solution exists

(Jacoby et al., 2015b,a). Therefore, Jacoby et al. (2015b) suggested an alternative method of parameters estimation, called the "bounded Generalized Least Squares method" (bGLS). The bGLS method formalises the serial dependence of asynchronies as a regression problem in which succeeding asynchronies linearly depend on previous asynchronies. 


$$
y=B x+Z,
$$

where

$$
y=\left|\begin{array}{c}
A_{1}-E\left[A_{k}\right] \\
\vdots \\
A_{N}-E\left[A_{k}\right]
\end{array}\right|, B=\left|\begin{array}{c}
A_{0}-E\left[A_{k}\right] \\
\vdots \\
A_{N-1}-E\left[A_{k}\right]
\end{array}\right|, x=(1-\alpha), Z=\left|\begin{array}{c}
H_{0} \\
\vdots \\
H_{N-1}
\end{array}\right|
$$

and where $A_{k}$ is the asynchrony at iteration $k=1, \ldots, N, N$ is the length of the sequence, $E\left[A_{k}\right]$ is the expected value of $A_{k}, \alpha$ is the correction coefficient. For this approach, $N$ should be the same for all sequences.

$Z$ follows a multivariate normal distribution with zero mean and $N$ x $N$ 130

variance-covariance matrix $\Sigma$. Considering that $Z=\left[Z_{0}, Z_{1}, \ldots, Z_{N-1}\right]^{T}$, where $Z_{k}=T_{k}+M_{k+1}-M_{k}-E\left[T_{k}\right]$, it can be specified by $\gamma_{Z}(j)=\operatorname{Cov}\left[Z_{k}, Z_{k+j}\right]$ according to

$$
\begin{aligned}
\gamma_{Z}(1) & =\operatorname{Cov}\left[\left(T_{k}+M_{k+1}-M_{k}\right),\left(T_{k+1}+M_{k+2}-M_{k+1}\right)\right] \\
& =\operatorname{Cov}\left[M_{k+1},-M_{k+1}\right] \\
& =-\sigma_{M}^{2} \\
\gamma_{Z}(0) & =\operatorname{Var}\left[T_{k}+M_{k+1}-M_{k}\right] \\
& =\sigma_{T}^{2}+2 \sigma_{M}^{2}, \\
\gamma_{Z}(j) & =0, j>1,
\end{aligned}
$$

so that

$$
\begin{aligned}
& Z \sim M V N(0, \Sigma), \Sigma=\gamma_{Z}(0) I+\gamma_{Z}(1) \Delta \\
& \gamma_{Z}(0)=2 \sigma_{M}^{2}+\sigma_{T}^{2}, \gamma_{Z}(1)=-\sigma_{M}^{2}, \gamma_{Z}(j)=0, j>1
\end{aligned}
$$




$$
\Delta=\left[\begin{array}{cccccc}
0 & 1 & 0 & \cdots & 0 & 0 \\
1 & 0 & 1 & \ddots & \vdots & 0 \\
0 & 1 & 0 & \ddots & 0 & \vdots \\
\vdots & 0 & \ddots & 0 & 1 & 0 \\
0 & \vdots & \ddots & 1 & 0 & 1 \\
0 & 0 & \cdots & 0 & 1 & 0
\end{array}\right] .
$$

$I$ is a $N \times N$ identity matrix and $\Delta$ is a $N$ x $N$ matrix determining non-zero correlations.

The $\log$-likelihood of $x$ and $\Sigma\left(\sigma_{T}, \sigma_{M}\right)$ give $Z(Z=y-B x)$ is

$$
l\left(x, \Sigma\left(\sigma_{T}, \sigma_{M}\right) \mid Z\right)=-\frac{N}{2} \log (2 \pi)-\frac{1}{2} \log (|\Sigma|)-\frac{1}{2}(y-B x)^{T} \Sigma^{-1}(y-B x) .
$$

Since $x$ and $\Sigma$ (i.e., $\alpha, \sigma_{T}$ and $\sigma_{M}$ ) are unknown, their estimation requires to iteratively estimate $x$ and $\Sigma$ referred to as feasible Generalized Least Squares (see Jung, 1987 in Repp, Keller \& Jacoby, 2012). This means that $x$ was estimated when $\Sigma$ was fixed at a particular (estimated) value and $\Sigma$ was estimated when $x$ was fixed at a particular (estimated) value. Because, the MLE estimator coincides with the GLS estimator, as noted by Jacoby et al. (2015b), the estimations could be performed by maximising $l(x, \Sigma \mid Z)$ of Equation 5 .

This parameter estimation method (bGLS) could be used on observations 45 from experiments with variable metronomes and it was generally less biased, more efficient, and faster than the traditional estimation techniques (Schulze \& Vorberg, 2002), for a wide range of settings (Jacoby et al., 2015b). In addition, the bGLS method could capture the synchronisation dynamics of two or more interacting individuals that coordinate in a group, as for instance when musical orchestra elements had to be coordinated (see also Wing, Endo, Bradbury \& Vorberg, 2014).

Yet, we identified three limitations of the presented methods: 
A) The presented methods require long asynchrony sequences. The more traditional methods used the acvf of the asynchrony sequence (Schulze \& Vorberg, 2002). For a meaningful acvf, it was suggested that the length should be at least $\min (N) \geq 50$ (Murteira et al., 1993). Similarly, the bGLS method searches for an approximated MLE. This is only reliable and unbiased if the sequence is relatively large (Ljung, 1998 in Jacoby, Keller, Repp, Ahissar \& Tishby, 2015a). As stated by Jacoby et al. (2015b), this should be at least $\min (N) \geq 30$.

But, in many natural situations that an experimenter might want to simulate, synchronisation can be observed for only very short time periods. In dance, partners alternately synchronise and eventually desynchronise their movements; in manufacturing work, the demand to coordinate with machines and other workers may be repetitive but short lasting; in a symphony orchestra, instruments such as cello, violin, piano, and celesta stand alone or together, and sometimes start and stop for very short time periods. A typical strategy in gait rehabilitation is that the patient synchronises the stepping pattern during walking with external cues (see e.g., Lim, van Wegen, de Goede, Deutekom, Nieuwboer, Willems, Jones, Rochester \& Kwakkel, 2005), but only for a few steps, probably in order to avoid fatigue. Short lasting interactions that involve movement synchronisation also exist in sports and in everyday coordination. These activities have in common that the movements become synchronised very quickly and last only short periods of time. Up to now, there do not seem to exist appropriate estimation approaches within the framework of event-based timing models, presented above, that can deal with short-lasting synchronisation phenomena.

B) Another important limitation of the methods is that they disregard information due to averaging. In a typical experimental paradigm, one makes inferences about parameters of the model that is supposed to have generated the behaviour. To achieve this, behavioural records are usually obtained repetitively, called trials or runs, and an average of these records, or their parameter estimates of each trial is taken.

Yet, by averaging, one may lose essential information, outliers can bias the 
results, and if trials with different lengths are included, they are weighted equally of the eLM to the bGLS in estimating parameters of the LPC model when the sequences are short. Then, we demonstrate the superior performance of the MEM to the eLM and the bGLS method, when estimating the parameters from 
sequences with varying intercepts.

\section{Methods}

\section{1. extended Linear Model (eLM)}

We developed the eLM based on Pinheiro \& Bates (2000). It is able to capture multiple sequences of asynchronies within a single model. Each asynchrony 220 is denoted by $a_{i j}, i=1, \ldots, m, j=1, \ldots, n_{i}$, where $i$ indexes the sequence and $j$ indexes the $j^{\text {th }}$ asynchrony within sequence $i$. The length of $a_{i}$ is denoted by $n_{i}$ and $N$ denotes here the length of all sequences together $N=\sum_{i=1}^{m} n_{i}$.

\subsubsection{Model}

The model can be written for each sequence $i$ as:

$$
\begin{aligned}
& y_{i}=x_{i} \beta+s_{i}, \\
& s_{i} \sim \operatorname{MVN}\left(0, \Sigma_{i}\right),
\end{aligned}
$$

225 where $y_{i}$ is a $\left(n_{i}-1\right) \times 1$ column vector of asynchronies of sequence $i, x_{i}$ is a $\left(n_{i}-1\right) \times 1$ column vector of asynchronies of sequence $i$ one iteration earlier than the asynchronies in vector $y_{i}, s_{i}$ is a $\left(n_{i}-1\right) \times 1$ column vector of the errors of sequence $i$, and $\Sigma_{i}$ is a $\left(n_{i}-1\right) \mathrm{x}\left(n_{i}-1\right)$ variance-covariance matrix

$$
\Sigma_{i}=\left[\begin{array}{cccccc}
\sigma_{T}^{2}+2 \sigma_{M}^{2} & -\sigma_{M}^{2} & 0 & \cdots & 0 & 0 \\
-\sigma_{M}^{2} & \sigma_{T}^{2}+2 \sigma_{M}^{2} & -\sigma_{M}^{2} & \ddots & \vdots & 0 \\
0 & -\sigma_{M}^{2} & \sigma_{T}^{2}+2 \sigma_{M}^{2} & \ddots & 0 & \vdots \\
\vdots & 0 & \ddots & \sigma_{T}^{2}+2 \sigma_{M}^{2} & -\sigma_{M}^{2} & 0 \\
0 & \vdots & \ddots & -\sigma_{M}^{2} & \sigma_{T}^{2}+2 \sigma_{M}^{2} & -\sigma_{M}^{2} \\
0 & 0 & \cdots & 0 & -\sigma_{M}^{2} & \sigma_{T}^{2}+2 \sigma_{M}^{2}
\end{array}\right] .
$$

The $s_{i}$ corresponds to $Z$ of the bGLS method: $s_{i}=$ ${ }_{230}\left[s_{i 1}, s_{i 2}, \ldots, s_{i n_{i}-1}\right]^{T}, s_{i j}=T_{i j}+M_{i j+1}-M_{i j}-E\left[T_{i j}\right]$. We changed its 
notation to prevent confusion with the random effects column vector $z$, introduced later. The model including all sequences is then

$$
Y=M V N(X \beta, \Sigma),
$$

where $Y$ and $X$ are column vectors with dimension $(N-m) \times 1$, and $\Sigma$ is a variance-covariance matrix with dimension $(N-m) \times(N-m)$

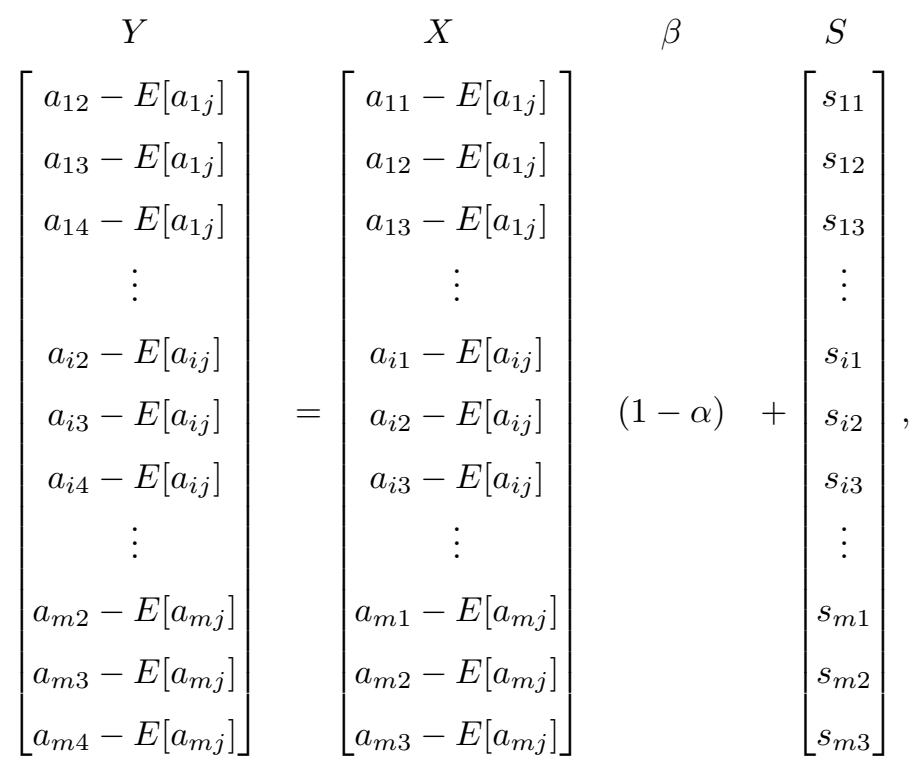

235 where $n_{i}=4, \forall i, i=1, \ldots, m$, for illustration purpose only.

\subsubsection{Computation}

The LPC model parameters $\alpha, \sigma_{T}$, and $\sigma_{M}$ can be obtained from $\beta$ and $\Sigma$. Based on the approach of Pinheiro \& Bates (2000), a single $\beta$ and $\Sigma$ can be estimated by a model including all sequences.

For computational reasons, $\sigma^{2}$ was factored out of $\Sigma_{i}$ :

$$
\frac{\Sigma_{i}}{\sigma^{2}}=\Lambda_{i}
$$


$\Lambda_{i}$ is parametrized by $\lambda$ :

$$
\Lambda_{i}=\left[\begin{array}{cccccc}
1 & \lambda & 0 & \cdots & 0 & 0 \\
\lambda & 1 & \lambda & \ddots & \vdots & 0 \\
0 & \lambda & 1 & \ddots & 0 & \vdots \\
\vdots & 0 & \ddots & 1 & \lambda & 0 \\
0 & \vdots & \ddots & \lambda & 1 & \lambda \\
0 & 0 & \cdots & 0 & \lambda & 1
\end{array}\right]
$$

Because it is a positive-definite matrix, it has an invertible square root $\Lambda_{i}^{\frac{1}{2}}$ so that $\Lambda_{i}=\left(\Lambda_{i}^{\frac{1}{2}}\right)^{T} \Lambda_{i}^{\frac{1}{2}}$. Then, $\Lambda_{i}^{-1}=\Lambda_{i}^{-\frac{1}{2}}\left(\Lambda_{i}^{-\frac{1}{2}}\right)^{T}$, where $\Lambda_{i}^{-\frac{1}{2}}$ is the inverse of $\Lambda_{i}^{\frac{1}{2}}$. The transformation to a linear model is then achieved by

$$
y_{i}^{*}=\left(\Lambda_{i}^{-\frac{1}{2}}\right)^{T} y_{i}, s_{i}^{*}=\left(\Lambda_{i}^{-\frac{1}{2}}\right)^{T} s_{i}, x_{i}^{*}=\left(\Lambda_{i}^{-\frac{1}{2}}\right)^{T} x_{i},
$$

which provides the linear model

$$
y_{i}^{*}=x_{i}^{*} \beta+s_{i}^{*},
$$

where $s_{i}^{*} \sim N V\left(\left(\Lambda_{i}^{-\frac{1}{2}}\right)^{T} 0, \sigma^{2}\left(\Lambda_{i}^{-\frac{1}{2}}\right)^{T} \Lambda_{i} \Lambda_{i}^{-\frac{1}{2}}\right)=N V\left(0, \sigma^{2} I_{i}\right)$.

For a fixed $\lambda$, the conditional MLEs are

$$
\begin{aligned}
& \hat{\beta}(\lambda)=\left(\left(X^{*}\right)^{T} X^{*}\right)^{-1}\left(X^{*}\right)^{T} Y^{*}, \\
& \hat{\sigma^{2}}(\lambda)=\frac{\left(Y^{*}-X^{*} \hat{\beta}\right)^{T}\left(Y^{*}-X^{*} \hat{\beta}\right)}{(N-m)},
\end{aligned}
$$

where $X=\left[X_{1}, \ldots, X_{m}\right]^{T}, Y=\left[Y_{1}, \ldots, Y_{m}\right]^{T}, \beta=(1-\alpha)$, and $N=\sum_{i}^{m} n_{i}$.

In the so called "profiled log-likelihood", $\beta$ can then be replaced by its conditional MLE so that $\beta$ is expressed as a function of $\lambda, \beta(\lambda)$. Therefore, the profiled log-likelihood is solely a function of $\lambda$ :

$l(\lambda \mid y)^{\text {profiled }}=\mathrm{const}-(N-m) \log \sqrt{\left(Y^{*}-X^{*} \hat{\beta}\right)^{T}\left(Y^{*}-X^{*} \hat{\beta}\right)}-\frac{1}{2} \sum_{i=1}^{m} \log \left|\Lambda_{i}\right|$. 
By optimising Equation 12 and using $\hat{\lambda}$ in Equation 11, the MLEs for $\hat{\beta}$ and $\hat{\sigma}^{2}$ can be computed. Subsequently, by using $\hat{\lambda}, \hat{\beta}$, and $\hat{\sigma}^{2}$, the final LPC model parameters are obtained by

$$
\begin{aligned}
& \hat{\alpha}=1-\hat{\beta}, \\
& \hat{\sigma}_{M}=\sqrt{-\hat{\sigma}^{2} \hat{\lambda}} \\
& \hat{\sigma}_{T}=\sqrt{\hat{\sigma}^{2}-2 \hat{\sigma}_{M}^{2}} .
\end{aligned}
$$

\subsection{Mixed-Effects Model (MEM)}

The MEM is an extension of the eLM that also incorporates random intercepts (Pinheiro \& Bates, 2000). It is able to capture multiple sequences of asynchronies within a single model and accounts for the between-sequence variability of the expected asynchrony of each sequence.

\subsubsection{Model}

The MEM is denoted as

$$
\begin{aligned}
y_{i} & =x_{i} \beta+z_{i} b_{i}+s_{i}, \\
b_{i} & \sim N V\left(0, \sigma_{b}^{2}\right), s_{i} \sim \operatorname{MVN}\left(0, \sigma^{2} \Lambda_{i}\right), i=1, \ldots, m,
\end{aligned}
$$

where $y_{i}, x_{i}$, and $s_{i}$ are defined as in Equation 6: $y_{i}$ is a $\left(n_{i}-1\right)$ x 1 column vector of asynchronies of sequence $i, x_{i}$ is a $\left(n_{i}-1\right)$ x 1 column vector of asynchronies of sequence $i$ one iteration earlier than the asynchronies in vector $y_{i}, s_{i}$ is a $\left(n_{i}-1\right) \times 1$ column vector of the errors of sequence $i$, and $\Lambda_{i}$ is a $\left(n_{i}-1\right) \times$ $\left(n_{i}-1\right)$ covariance matrix.

The $b_{i}$ is a $m \times 1$ column vector of random effects for sequence $i$ and $z_{i}$ is a $\left(n_{i}-1\right) \times m$ design matrix, indexing $b_{i}$. The $b_{i}$ is normally distributed with zero mean and (the scalar) standard deviation $\sigma_{b}$. It represents the variability between the expected asynchrony values $E\left[a_{i j}\right]$ among the sequences. The $b_{i}$ and $s_{i}$ are independent within and between sequences. 


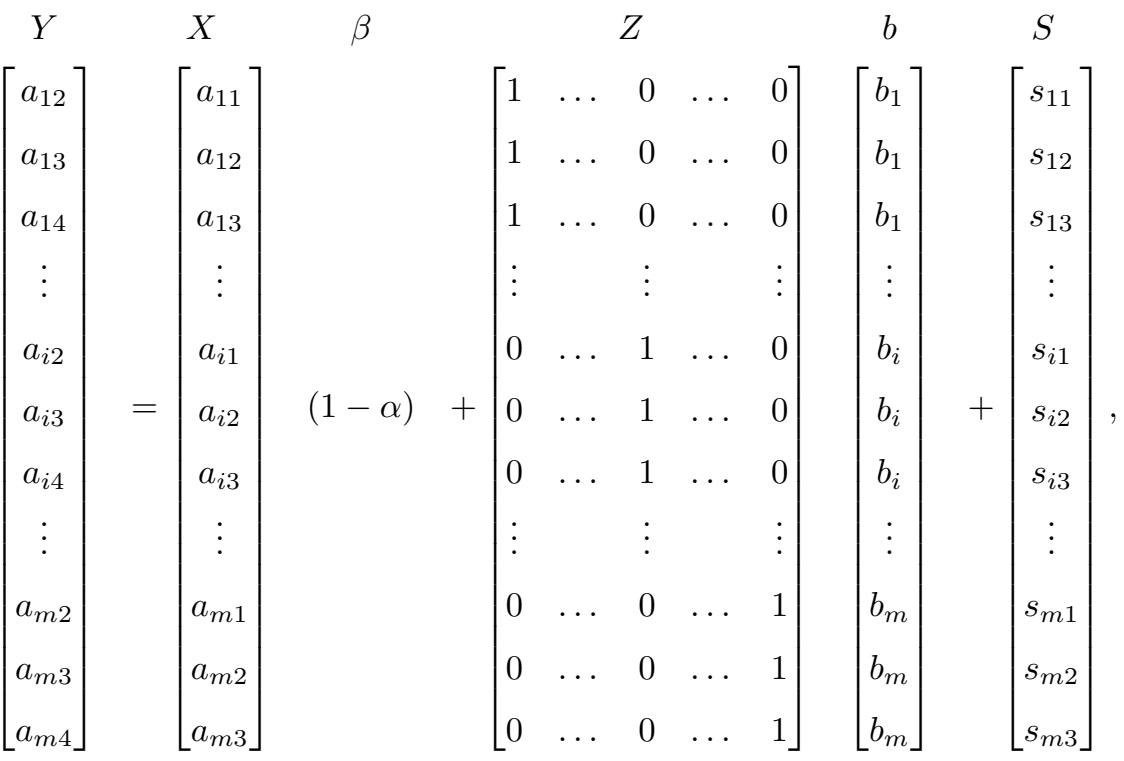

where $n_{i}=4, \forall i, i=1, \ldots, m$, for illustration purpose only.

\subsubsection{Computation}

In order to obtain the parameters $\beta, \sigma_{b}$, and $\sigma^{2}$, the following likelihood function can be maximised:

$L\left(\beta, \delta, \sigma^{2} \mid y\right)=\frac{1}{\left(2 \pi \sigma^{2}\right)^{(N-m) / 2}} \exp \left(\frac{-\sum_{i=1}^{m}\left\|\tilde{y}_{i}-\tilde{x}_{i} \beta-\tilde{z}_{i} \hat{b}_{i}\right\|^{2}}{2 \sigma^{2}}\right) \prod_{i=1}^{m} \frac{|\delta|}{\sqrt{\left|\tilde{z}_{i}^{T} \tilde{z}_{i}\right|}}$,

where $\delta$ parametrises the variance-covariance matrix of the random effects $b_{i}$

(which is here a scalar $\sigma_{b}$ ), $\sigma$ is the residual standard error of $s_{i}, \tilde{y}_{i}, \tilde{x}_{i}$, and $\tilde{z}_{i}$ are the augmented data vectors

$$
\tilde{y}_{i}=\left[\begin{array}{c}
y_{i} \\
0
\end{array}\right], \tilde{x}_{i}=\left[\begin{array}{c}
x_{i} \\
0
\end{array}\right], \tilde{z}_{i}=\left[\begin{array}{c}
z_{i} \\
\delta
\end{array}\right], \delta=\sqrt{\frac{\sigma^{2}}{\sigma_{b}^{2}}},
$$

and $\hat{b}_{i}$ is estimated by:

$$
\hat{b}_{i}=\left(\tilde{z}_{i}^{T} \tilde{z}_{i}\right)^{-1} \tilde{z}_{i}^{T}\left(\tilde{y}_{i}-\tilde{x}_{i} \beta\right), i=1 \ldots, m .
$$


Since the OLS for $\hat{b}_{i}$ depends on $\beta$, and the OLS for $\hat{\beta}$ depends on $b_{i}$, they must be estimated jointly (iteratively).

However, because the within-sequence errors are correlated, it was performed a linear transformation of the variables, as previously (see Equation 9):

$$
y_{i}^{*}=\left(\Lambda_{i}^{-\frac{1}{2}}\right)^{T} y_{i}, s_{i}^{*}=\left(\Lambda_{i}^{-\frac{1}{2}}\right)^{T} s_{i}, x_{i}^{*}=\left(\Lambda_{i}^{-\frac{1}{2}}\right)^{T} x_{i}, z_{i}^{*}=\left(\Lambda_{i}^{-\frac{1}{2}}\right)^{T} z_{i},
$$

which provided the linear Mixed-Effects Model

$$
\begin{aligned}
& y_{i}^{*}=x_{i}^{*} \beta+z_{i}^{*} b_{i}+s_{i}^{*}, \\
& b_{i} \sim N V\left(0, \sigma_{b}^{2}\right), s_{i}^{*} \sim M V N\left(0, \sigma^{2} I\right), i=1, \ldots, m .
\end{aligned}
$$

Its profiled likelihood function can be expressed as

$$
L\left(\beta, \delta, \sigma^{2}, \lambda \mid y\right)^{\text {profiled }}=L\left(\beta, \delta, \sigma^{2}, \lambda \mid y^{*}\right) \prod_{i=1}^{m}\left|\Lambda_{i}^{-1 / 2}\right|,
$$

where $\lambda$ parametrises $\Lambda_{i}$, as in Equation 12. By optimising Equation 20, its best fitting parameters can be obtained from which $\alpha, \sigma_{T}$, and $\sigma_{M}$ were computed. For a detailed description of the proof and most efficient computation of $L\left(\beta, \delta, \sigma^{2}, \lambda \mid y\right)^{\text {profiled }}$, see Pinheiro \& Bates (2000).

\section{Results \& Discussion}

In order to evaluate and compare the performance of different methods, we simulated asynchrony sequences that could be the output of an experiment using SMS tasks. This was done by running the LPC model in Equation 2. It received as input a) an initial asynchrony $A_{1}$, sampled from $\operatorname{NV}(0,20)$, b) a constant number of metronome events $n$, and c) a set of parameters $\left(\sigma_{T}, \sigma_{M}, \alpha\right)$. So, this model was iterated $n$ times. Parameter settings were held close to those of previous studies (Jacoby et al., 2015b). The output of a single simulation was a sequence of asynchronies of length $n$. Similar to Jacoby et al. (2015b), one parameter estimate was based on 15 of such sequences. For validation, we considered 50 estimates. Therefore, the LPC model was simulated $15 x 50=450$ 
times and its mean estimate and the $95 \%$ confidence interval were computed from the 50 estimates. The parameter estimation methods were evaluated by considering its accuracy and efficiency with which they recovered the set of parameters of the LPC model that had generated the data.

\section{1. eLM and sequence length}

In order to compare the eLM with the bGLS, the asynchrony sequences were simulated with different lengths $(n=5, n=10, n=30)$. Results revealed that the eLM is less biased for different sequences lengths compared to the bGLS method (see Figure 1). While the bias of the bGLS method increased with decreasing sequence length and the size of the confidence intervals remained very similar, the eLM seems unbiased at any length but increased the confidence intervals.

This was expectable, considering that the bGLS method averaged estimates from single sequences. When estimating the parameters by approximating an MLE from a short sequence, estimations can fail easily, which results in estimates that may consistently deviate from the theoretical mean. In addition, mean estimates are very susceptible to outliers. Thus, when there are few asynchronies, bGLS estimates can be biased.

In contrast, in the eLM method, a single parameter $\hat{\lambda}$ is estimated by maximizing a profiled MLE (see Equation 12) involving all indexed sequences. Afterwards, two single parameters $\hat{\beta}(\lambda)$ and $\hat{\sigma}(\lambda)$ can be estimated by the conditional MLEs (see Equation 11) and a simple transformation reveals then the final parameter estimates of the LPC model $\hat{\alpha}, \hat{\sigma}_{M}$, and $\hat{\sigma}_{T}$ (see Equation 13). This method employs every single sequence for parameter estimation while other sequences provide additional information about "what is going on" in the particular sequence. This makes the eLM method more resistant to estimation biases. However, short sequences should still lead to less efficient estimations. This is here reflected by an increase of the confidence intervals.

Notwithstanding, the confidence intervals in the eLM are particularly large 
Comparison of bGLS and eLM on asynchrony sequences with different lengths
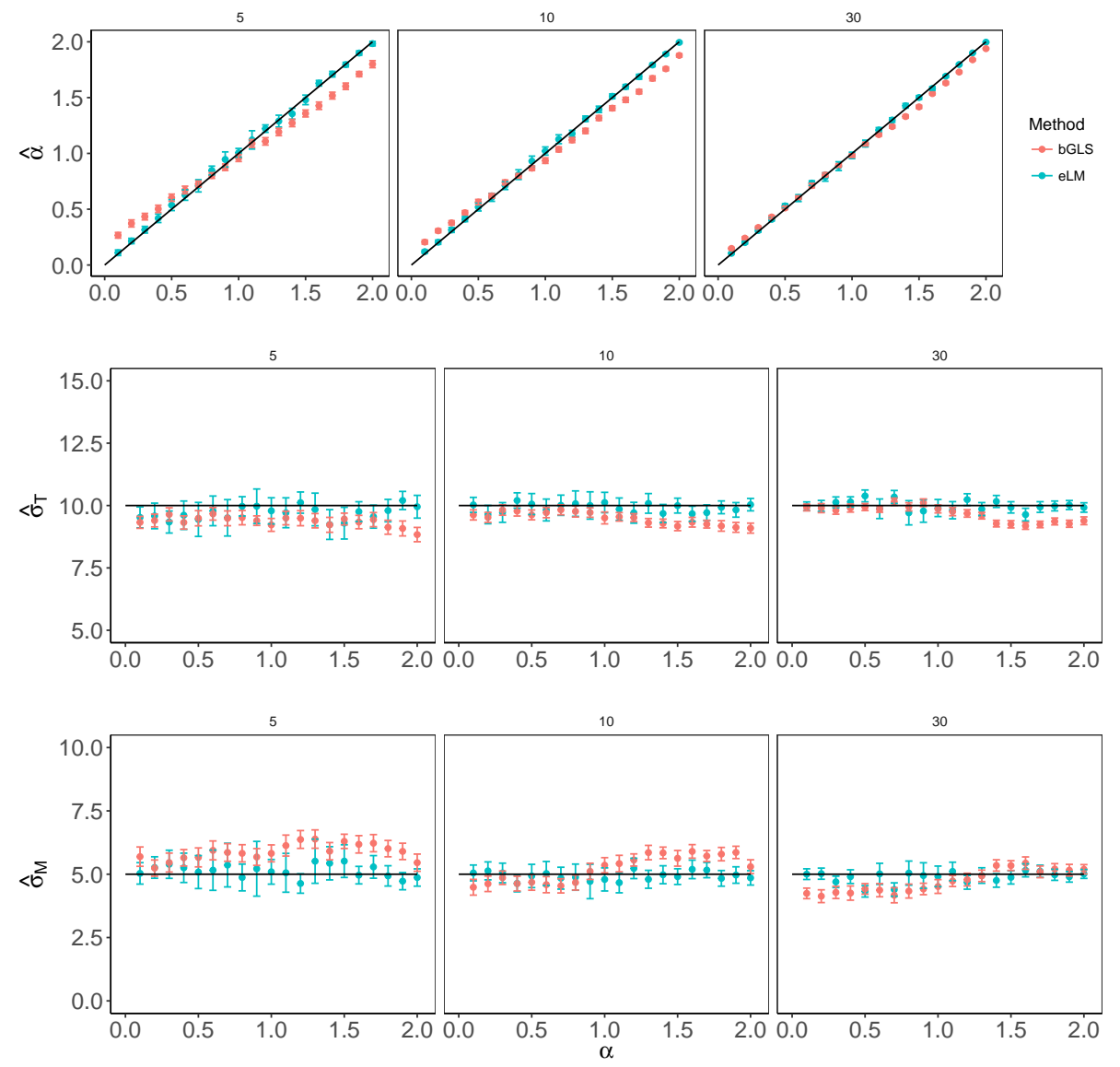

Figure 1: Comparison of the LPC model parameter estimation methods as a function of true $\alpha$ for different sequence lengths. The plots show the mean and the $95 \%$ confidence intervals of 50 estimates for each $\alpha$. The estimates of the bGLS method are displayed in red and the estimates of the eLM method are displayed in magenta. Dots are the mean estimates. When lying on the black line, the estimates coincide with the true values. The sequences were obtained by simulating the LPC model with $\sigma_{M}=5, \sigma_{T}=10$ and $\alpha$ according to the ordinate. Each estimate was obtained from $m=15$ asynchronies sequences with varying length $\left(n_{i}=30, n_{i}=10, n_{i}=5, \forall i, i=1, \ldots, 15\right)$. 
Jacoby, Keller, Repp, Ahissar \& Tishby, 2015a). In future studies, additional bounding conditions could be included and evaluated for the eLM method.

Concluding, the eLM method seems to exploit a trade-off between accuracy (mean deviation from the "true" parameter) and efficiency (variability of the estimations) in favour of the former. When uncertainty increases due to less information within the empirical observations, its efficiency decreased in order to hold a high accuracy.

A shortcoming of the eLM method and the bGLS method is that they presume the same parameter settings among all sequences. Apparently, proposing a general model, like the LPC, only has value if the process in question is stable in its parameter settings, as long as the environment is constant. However, when the asynchronies were obtained from experiments, there should be variability that is not related to the LPC model. For instances, in a repeated measurement design, identical experimental conditions among trials are impossible to achieve. When there are noise factors that are independent among all asynchronies, the variability is captured by the error term $(\epsilon)$ of the model. Yet, there may be factors that have a unique contribution on each trial and are hard to control. This leads to variability between trials that is neither accounted for by the LPC model nor by the introduced parameter estimation methods.

345 Concretely, we have shown that the eLM is appropriate if the mean asynchrony is expected to be constant among sequences. Jacoby et al. (2015b) normalised the asynchronies by the mean asynchrony obtained from so many asynchrony records as possible. However, if each sequence is exposed to factors that contribute uniquely to each sequence, such a normalisation is inappropriate. Another possibility is to normalise each sequence by the mean asynchrony of the respective sequence. This can be done when using the eLM and the bGLS method. Yet, for short sequences, such mean asynchrony might not be very representative.

One possible solution to account for the variability would be to incorporate 355 another fixed effect in the eLM, with as many parameters as there are sample means. Considering that this value was sampled from a continuous distribution, 
the number of parameters would equal the number of sequences $i=1, \ldots, m$. But, we were not interested in making inferences about the specific effects of these "noise factors" but must control them for an unbiased estimation of the LPC model parameters. For this reason, a solution is to account for varying $\mu_{a_{i}}$ among sequences $i$ by incorporating random-effects (random intercepts) in the eLM model, making it a single-level MEM. This requires the estimation of far fewer parameters than when using fixed effects, and it seems theoretically more plausible.

\subsection{MEM and between-sequence variability}

In order to compare the MEM with the eLM and the bGLS method, we simulated asynchrony sequences with different magnitudes of the between-sequence variability of the intercept (i.e., the expected asynchronies varied between sequences). The results revealed that the bGLS method and the eLM method deteriorated with increasing between-sequence variability. The here presented eLM method normalised the sequences by the sample mean of each sequence and the bGLS method computed the sample mean by all asynchronies from all sequences. The biases increased with between-sequence variability and were smaller for lower $\alpha$. The patterns of these biases are quite complex and we do 375 yet not know how to interpret them.

In contrast, the MEM method, which modelled the between-sequence variability by random intercepts, led to unbiased estimates for different magnitudes of variability (see Figure 2). We further benchmarked the functions showing that the most complex MEM method is slightly faster than the bGLS method $380 \quad(\mathrm{MEM}=15 \mathrm{~ns}, \mathrm{bGLS}=20 \mathrm{~ns})$. We, therefore, suggest that the MEM method is an appropriate alternative that can be used for single sequences when they are sufficiently long and stationary, and for short and multiple sequences when they are stationary. 

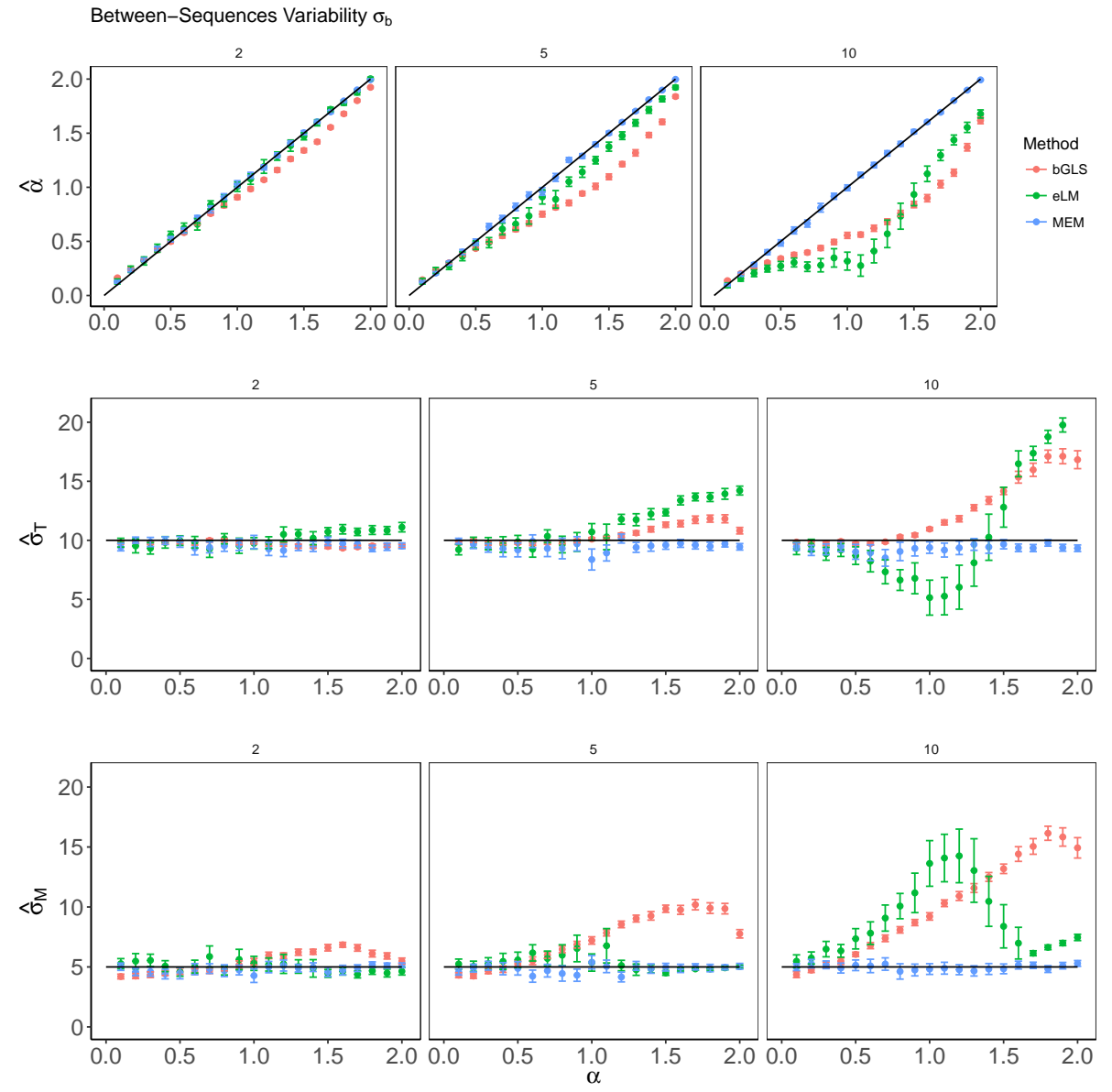

Figure 2: Comparison of the bGLS, eLM, and MEM when there is additional between-sequence variability of $E\left[a_{i j}\right]$ that is not incorporated in the LPC model. These random intercepts were sampled from $N V\left(0, \sigma_{b}^{2}\right), \sigma_{b}=2,5,10$, as indicated at the top of the plots. The parameters were estimated for different $\alpha$ from sequences of length $n_{i}=30$. The sequences were obtained by simulating the LPC model with $\sigma_{T}=10$ and $\sigma_{M}=5$. 


\section{General Discussion}

385

The main goal of this work was to introduce unbiased methods of parameter estimation of the LPC model. Therefore, we simulated asynchrony sequences from the LPC model, replicated the current "state of art" estimation method (bGLS), and compared this with two here-developed methods in conditions that often occur in experimental setups.

\subsection{Contributions of the extended Linear Model (eLM)}

We demonstrated that the bGLS is prone to bias when the asynchrony sequences become shorter. We suggest that this owes to the inefficient technique of averaging parameter estimates, particularly when there is little information on each sequence. For this reason, we presented eLM, which integrates multiple sequences into a single model. Our results revealed that eLM estimates the model parameters of longer and shorter sequences with less bias than the bGLS.

Besides the simultaneous consideration of multiple sequences, another advantage of such an approach is that it can deal with balanced and unbalanced data. This implies that sequences of different lengths and/or missing values can be included. In contrast to the bGLS method, the eLM weights stronger the sequences with greater lengths, what is appropriate because longer sequences include more information and lead to less biased estimates. As far as we know, the authors of the bGLS method did not address this issue. We assume that shorter sequences or sequences with missing values were disregarded.

\subsection{Contributions of the Mixed-Effects Model (MEM)}

The MEM is an extension of the eLM and also considers the betweensequence variability of the expected asynchrony within each sequence. By relating random effects to the asynchronies sharing the same sequence, the MEM could flexibly account for this variability. It provided unbiased estimates where the bGLS and the eLM methods largely deteriorated.

For the simulation, we produced between-sequence variability by adding a value, sampled from $N V\left(0, \sigma_{b}^{2}\right)$, with different $\sigma_{b}^{2}$, which was invariable within 
a sequence but variable between sequences. How could one justify the validity of this manipulation?

When the different sequences result from repeated measurements on the same individual, such between-sequence variability might reside from an interplay of physiological factors - the properties of the individual sensory systemand psychological factors - for instances attentional focus and distraction. In order to achieve synchronisation, the asynchronies have to be perceived as such. The perception of asynchrony depends on a complex interaction of a multitude of sensory cues from various modalities (e.g., tactile, auditory, \& visual). Different sensory systems vary in propagation, transduction, transmission, and processing times, leading to different magnitudes of the physical (actual) asynchronies when an environmental event is represented by multiple modalities (see 425 e.g., Noy, 2018).

The attentional focus of the individual on a particular sensory cue determines the size of the asynchrony that is required to be perceived as synchronous. Thus, attention might affect the information processing delays represented by the parameter $\mu_{M}$ in the LPC model. Consequently, the individual might attempt to achieve and stabilise different asynchronies, resulting in different mean asynchronies among sequences.

Yet, it is not clear why the attentional focus should vary between sequences and not within a sequence. In a typical synchronisation experiment, event sequences are presented visually on a computer screen or aurally through headphones and suddenly appear and disappear. Before a sequence is presented, the participant's attention is purposefully caught by the presentation of, for instance, a visual fixation cross or a beep sound. Individual sequences are usually separated by short time periods. During stimulus presentation within a trial (e.g., a sequence of 10 to 30 metronome clicks), an individual should be able to 440 stay focused and remains within a similar cognitive state. However, completing the task may require $15 \mathrm{~min}$ to $120 \mathrm{~min}$; time enough for the individual's mind to wander and to focus different stimulus attributes. Although these issues should be approached in further studies, we believe the presence of attentional shifts 
during such paradigms can produce between-sequence variability by affecting information processing delays.

When sequences are the performance of different individuals, then the between-sequence variability could owe to factors specific to each individual. This is, for example, the individual's focus of attention. But also, the parameter settings of the underlying LPC model (e.g., perceptional delays and motor delays) should be different among individuals. Such individual differences produce very large between-sequence variations.

While the former parameter estimation methods cannot be used when each sequence is the performance of a different participant, the MEM approach can be implemented. A possible application would be to assess the effects of particular experimental conditions - for example, some stimulus properties - on general timing parameters. Then, one is not interested in making inferences about the differences of the LPC parameters among individuals, but still, has to control them in order to achieve unbiased estimates. This can be done by incorporating random effects on the individual level, as illustrated by the present work.

Finally, another variability factor that could be controlled by the MEM is methodological. Variability between sequences could result from the stimuluspresenting or the performance-capturing systems.

Concluding, we presented several examples that emphasise that it is highly relevant to include random intercept parameters into methods that estimate 465 the parameters of the LPC model from experimental data. Here, we simulated sequences with between-sequence variability and assessed a model with a single random intercept but such a model could also incorporate multiple and nested random-effects.

\subsection{Limitations}

It must be mentioned that the eLM and the MEM approach estimated the parameters by maximising likelihood functions. Alternatively, one could have used restricted maximum likelihood functions, which are generally more robust since they consider the number of degrees of freedom (Pinheiro \& Bates, 2000). 
In order to test this, we compared both functions by using the same observations and we did not observe any significant differences. It is known that both can be maximised to estimate the same fixed effects and that the likelihood estimate is unbiased for large overall sample sizes (Pinheiro \& Bates, 2000). This is usually the case in experimental setups. Nevertheless, future studies should approach this question by estimating the parameters with both functions while systematically manipulating the size of the sample.

A limitation might be that the presented methods require stationary asynchronies. This is difficult to assure for short synchronisation periods taking into account that synchronisation might be a highly transient process (see e.g., van Ulzen, Lamoth, Daffertshofer, Semin \& Beek, 2008). Nevertheless, stationary asynchronies are an important requirement of the LPC model and we suggest that non-stationarity should be prevented by cautiously designing the experiments and preparing the data set for analysis, rather than being modelled explicitly.

Another limitation of this work might be that between-sequence variability values were chosen without being externally validated. For the LPC model parameter settings, we could use settings similar to previous studies (Jacoby et al., 2015b; Schulze \& Vorberg, 2002). For the between-sequence variability, however, we have chosen values based on several tests and theoretical plausibility. Future studies should address this question and actually quantify the between-sequence variability that occurs in SMS tasks.

Related to the previous limitations, the here developed methods were validated on simulated asynchrony sequences. The next step should be to validate the methods on observations obtained from experiments.

Finally, our work was strictly concerned with the LPC model. The principal assumption of the LPC model is that corrections are performed on the perceived deviations from the participants' taps from the corresponding stimulus event onset. Surely, this is a quite simplistic model of reality since it presumes that even highly small asynchronies are registered by the individual. There are plenty of studies showing that asynchronies falling into a temporal integration window 
(see Vroomen \& Keetels, 2010). It would be interesting to evaluate this model regarding the inquiry of asynchrony thresholds for awareness, phase, and period correction, etc. (see Repp, 2005).

Nevertheless, this work does not address the plausibility of the LPC model to increase the applicability of the model to more complex settings. Moreover, motivated by parsimony as a fundamental principle for developing models, the LPC still finds great use in basic and applied research (see Jacoby, Tishby, Repp, Ahissar \& Keller, 2015b).

\subsection{Further Contributions}

One advantage of the approaches here developed is the existence of validated software for fitting the eLM and the MEM, namely the "nlme" and the "lmer" R-packages. Their use requires a different parametrization of the variance-covariance matrix, but, besides being more robust, they are also quicker than the bGLS method.

Moreover, in order to examine the different parameter estimation methods, we translated the Matlab code provided by Jacoby et al. (2015b) into R code and adjusted it for the particular question. We also implemented computational methods presented in Pinheiro \& Bates (2000), in order to flexibly modify the 525 Mixed-Effects model structure for the purpose of our study. All programs ( $R$ codes) developed for this study are available on GitHub (2017).

\subsection{Conclusions}

In sum, we provided a general framework of Mixed-Effects Models to estimate the parameters of the LPC model. We do not claim for the overall validity of the LPC. A more profound exploration of the LPC applicability to a large scope of natural settings is outside the scope of this work. Nevertheless, we demonstrated that Mixed-Effects Models are highly useful for achieving unbiased and efficient parameter estimations of the LPC from synchronisation performances in SMS tasks. It remains to explore the extension of these methods, 

transition, and non-stationary asynchronies.

\section{References}

GitHub (2017). Github. https://github.com.

Grush, R. (2004). The emulation theory of representation: Motor control, imagery, and perception. Behavioral and brain sciences, 27, 377-396.

Jacoby, N., Keller, P. E., Repp, B. H., Ahissar, M., \& Tishby, N. (2015a). Lower bound on the accuracy of parameter estimation methods for linear sensorimotor synchronization models. Timing $\&$ Time Perception, 3, 32-51.

Jacoby, N., Tishby, N., Repp, B. H., Ahissar, M., \& Keller, P. E. (2015b).

Lim, I., van Wegen, E., de Goede, C., Deutekom, M., Nieuwboer, A., Willems, A., Jones, D., Rochester, L., \& Kwakkel, G. (2005). Effects of external rhythmical cueing on gait in patients with parkinson's disease: a systematic review. Clinical rehabilitation, 19, 695-713.

Miles, L. K., Nind, L. K., \& Macrae, C. N. (2009). The rhythm of rapport: Interpersonal synchrony and social perception. Journal of experimental social psychology, 45, 585-589.

Mörtl, A., Lorenz, T., Vlaskamp, B. N., Gusrialdi, A., Schubö, A., \& Hirche, S. (2012). Modeling inter-human movement coordination: synchronization 
Murteira, B. F., Muller, D. A., \& Turkman, K. F. (1993). Analise de sucessoes cronologicas. McGraw-Hill, Lisboa.

Myung, I. J. (2003). Tutorial on maximum likelihood estimation. Journal of mathematical Psychology, 47, 90-100.

Noy, D. (2018). A multimodal approach to interpersonal gait synchronization.. Ph.D. thesis Uminho. Unpublished thesis.

Noy, D., Mouta, S., Lamas, J., Basso, D., Silva, C., \& Santos, J. A. (2017). Audiovisual integration increases the step synchronization of side-by-side walkers. Human Movement Science, .

570 Pinheiro, J., \& Bates, D. (2000). Mixed-effects models in S and S-PLUS. Springer, New York.

Reddish, P., Fischer, R., \& Bulbulia, J. (2013). Lets dance together: synchrony, shared intentionality and cooperation. PloS one, 8, e71182.

Repp, B. H. (2001). Processes underlying adaptation to tempo changes in sensorimotor synchronization. Human movement science, 20, 277-312.

Repp, B. H. (2005). Sensorimotor synchronization: a review of the tapping literature. Psychonomic bulletin \& review, 12, 969-992.

Repp, B. H., \& Keller, P. E. (2004). Adaptation to tempo changes in sensorimotor synchronization: Effects of intention, attention, and awareness. Quarterly Journal of Experimental Psychology Section A, 57, 499-521.

Repp, B. H., \& Keller, P. E. (2008). Sensorimotor synchronization with adaptively timed sequences. Human movement science, 27, 423-456.

Repp, B. H., Keller, P. E., \& Jacoby, N. (2012). Quantifying phase correction in sensorimotor synchronization: empirical comparison of three paradigms. Acta psychologica, 139, 281-290. 
Schmidt, R. C., \& Richardson, M. J. (2008). Dynamics of interpersonal coordination. In Coordination: Neural, behavioral and social dynamics (pp. 281-308). Springer.

Schulze, H.-H., \& Vorberg, D. (2002). Linear phase correction models for synchronization: Parameter identification and estimation of parameters. Brain and Cognition, 48, 80-97.

Torre, K., \& Balasubramaniam, R. (2009). Two different processes for sensorimotor synchronization in continuous and discontinuous rhythmic movements. Experimental Brain Research, 199, 157-166.

van Ulzen, N. R., Lamoth, C. J., Daffertshofer, A., Semin, G. R., \& Beek, P. J. (2008). Characteristics of instructed and uninstructed interpersonal coordination while walking side-by-side. Neuroscience Letters, 432, 88-93.

Valdesolo, P., Ouyang, J., \& DeSteno, D. (2010). The rhythm of joint action: Synchrony promotes cooperative ability. Journal of Experimental Social Psychology, 46, 693-695.

Valturio, R. (1921). De re militari. Wechel.

Van Der Steen, M. C., \& Keller, P. E. (2013). The adaptation and anticipation model (adam) of sensorimotor synchronization. Frontiers in human neuroscience, 7, 253.

Vroomen, J., \& Keetels, M. (2010). Perception of intersensory synchrony: a tutorial review. Attention, Perception, \&3 Psychophysics, 72, 871-884.

Wiltermuth, S. S., \& Heath, C. (2009). Synchrony and cooperation. Psychological science, 20, 1-5.

Wing, A. M., Endo, S., Bradbury, A., \& Vorberg, D. (2014). Optimal feedback 610 correction in string quartet synchronization. Journal of The Royal Society Interface, 11, 20131125. 
Wing, A. M., \& Kristofferson, A. B. (1973). Response delays and the timing of discrete motor responses. Perception \& Psychophysics, 14, 5-12.

Zelaznik, H. N., Spencer, R., \& Ivry, R. B. (2002). Dissociation of explicit and implicit timing in repetitive tapping and drawing movements. Journal of Experimental Psychology: Human Perception and Performance, 28, 575. 